\title{
THE GALOIS CORRESPONDENCE FOR LINEAR HOMOGENEOUS DIFFERENCE EQUATIONS
}

\author{
CHARLES H. FRANKE
}

1. Summary. In general the notation and terminology are as in [1]. We assume the following throughout. All fields are inversive difference fields of characteristic zero. If $N$ is a difference field then $C_{N}$ is the subfield of $N$ of constants, (solutions to $y_{1}=y$ ). $M$ is the field obtained from a field $k$ by adjoining a fundamental system $a$ $=\left(a^{(1)}, \cdots, a^{(n)}\right)$ for a linear homogeneous difference equation of effective order $n$ in $k\{y\} . K$ is the algebraic closure in $M$ of $k\left(C_{M}\right)$ and $G$ is the transformal Galois group of $M$ over $K . L$ is a difference subfield of $M$ containing $K$, and $D$ is the transformal Galois group of $L$ over $K$.

A partial Galois correspondence for $M$ as an extension of $K$ can be deduced from [2, Theorem 4] and [3, Theorem]. In this note the correspondence is completed by showing that a connected normal subgroup $H$ of $G$ corresponds to a relatively closed intermediate field $L$ which is normal over $K$, and conversely. Further, when this situation exists, $G / H$ is dense, in a natural topology, in $D$. An example is given where $G / H$ is not naturally isomorphic to all of $D$.

Topological statements will refer to the Zariski topology on algebraic sets and to the topology it induces on factor groups of algebraic matrix groups.

Some familiarity with [2, p. 491-499] will be assumed.

2. Universal extensions. $N$ is a universal extension of $M$ for $L$ if $N$ is a difference overfield of $M$ and every difference automorphism of $L$ over $K$ can be extended to a difference isomorphism of $M$ into $N$. Example 4.2 below shows that even if $L$ is itself a solution field over $K, M$ need not be a universal extension for $L$.

Proposition 2.1. If $L$ is relatively closed in $M$ then universal extensions of $M$ for $L$ exist.

Proof. To avoid set-theoretic difficulties in the application of Zorn's Lemma we choose an algebraically closed field $U$ so that the transcendence degree of $U$ over $M$ is the second infinite cardinal greater than the cardinal of $D$. Contrary to our usual convention we do not assume that $U$ is a difference field. Consider the set of pairs $\left(D^{\prime}, N^{\prime}\right)$ with the following properties. (1) $D^{\prime} \subseteq D$. (2) $N^{\prime}$ is a differ-

Received by the editors May 17, 1968. 
ence overfield of $M$ and the underlying algebraic field of $N^{\prime}$ is contained in $U$. (3) Each $S \in D^{\prime}$ extends to a difference isomorphism $\bar{S}$ of $M$ into $N^{\prime}$ and $N^{\prime}$ is generated over $M$ by the images $\bar{S}(M)$.

If the pairs are ordered by inclusion then the set is clearly inductive. If $\left(D^{*}, N\right)$ is a maximal element and $T \in D$ then $T$ is a difference isomorphism of $L$ into $N$. Since $L$ is relatively closed in $M, T$ extends to a difference isomorphism of $M$ into a difference overfield $N^{\prime}$ of $N$ [1, Chapter 9, Theorem 1].

Since $M$ is finitely generated over $L$, the transcendence degree of $N$ over $M$ is at most the first infinite cardinal greater than the cardinal of $D$. Therefore $N^{\prime}$ can be chosen so that the underlying algebraic field of $N^{\prime}$ is a subset of $U$. Then, since $\left(D^{*}, N\right)$ is maximal, $T \in D^{*}$, $D^{*}=D$ and $N$ is a universal extension of $M$ for $L$.

3. Isomorphism groups. If $N$ is a fixed difference overfield of $M$ then $I_{L}$ will denote the set of difference isomorphisms of $M$ into $N$ leaving $L$ fixed. $I_{K}$ will be denoted by $I$.

Proposition 3.1. If $L$ is relatively closed in $M$ then $I_{L}$ is a connected algebraic matrix group. The Galois group $G_{L}$ of $M$ over $L$ is dense in $I_{L}$.

Proof. Let $P$ be the set of all polynomials in $C_{K}[x], x=\left(x^{(i, j)}\right), i, j$ $=1, \cdots, n$, which vanish on the matrices of the elements of the Galois group $G_{L}$. Then $P$ is a prime ideal of dimension t.d. $(M, L)$, and $I_{L}$ is in one to one correspondence with the set of nonsingular solutions to $P$ in $C_{N}$ [2, Theorems 1 and 2] and [3, p. 549]. If $C^{*}$ is the algebraic closure of $C_{K}$ then the ideal $U$ generated by $P$ in $C^{*}[x]$ is prime $\left[3\right.$, p. $\left.549-550, U=\bar{S}^{*}\right]$. Therefore the ideal generated by $P$ in $\left(C^{*} \cap C_{N}\right)[x]$ is prime. Finally, the ideal generated by $P$ in $C_{N}[x]$ is prime [1, Introduction, Theorem 23].

Let $Q$ be the set of all polynomials in $C_{N}[x]$ which vanish on $I_{L}$. Each $F \in Q$ can be written in the form $\sum v_{i} F^{(i)}$ where $F^{(i)} \in C_{K}[x]$ and $v_{i}$ is a vector space basis of $C_{N}$ over $C_{K}$. Since $F$ is zero for each matrix of an element in $G_{L}$ the same is true for each $F^{(i)}$. Therefore $F^{(i)} \in P, Q$ is the ideal generated by $P$ in $C_{N}[x]$ and $Q$ is prime. Since $\operatorname{dim} G_{L}=\operatorname{dim} I_{L}, G_{L}$ is dense in $I_{L}$.

To complete the proof it is sufficient to show that $I_{L}$ is a group under an operation corresponding to the multiplication of associated matrices.

If $S \in I_{L}$ then, since $L\left(C_{N}\right)$ and $M$ are linearly disjoint over $L$, [2, Proposition 1], $S$ extends uniquely to a difference isomorphism $\bar{S}$ of $M\left(C_{N}\right)$ over $L\left(C_{N}\right)$. Since $S\left(a^{(j)}\right) \in M\left(C_{N}\right)$ for each $j, \bar{S}$ maps $M\left(C_{N}\right)$ into itself. If the matrix of $S$ is $\left(c_{i j}\right)$, then the equations $S\left(a^{(j)}\right)=\sum c_{i j} a^{(i)}$ 
can be solved for the $a^{(i)}$ as a linear combination of the $S\left(a^{(i)}\right)$ with coefficients in $C_{N}$. Therefore $\bar{S}$ is a difference automorphism of $M\left(C_{N}\right)$. We define the product of $S, T \in I_{L}$ as the composition $\bar{S} T$, and the group inverse of $S$ as the restriction of $\bar{S}^{-1}$ to $M$. Under these operations $I_{L}$ is a group isomorphic to the set of matrices corresponding to the elements of $I_{L}$ and the proof is complete.

Corollary 3.2. $I_{L}$ is isomorphic to the Galois group $G^{*}$ of $M\left(C_{N}\right)$ over $L\left(C_{N}\right)$.

Proof. $I_{L}$ and $G^{*}$ are each isomorphic to the same group of matrices.

4. The Galois correspondence. The notation is as above with primes denoting the Galois correspondence.

TheOREM 4.1. Assume that $H$ is an algebraic subgroup of $G$ and that $L$ is an intermediate field.

(1) $H^{\prime \prime}=H$.

(2) If $L$ is relatively closed then $L^{\prime \prime}=L$, and $M$ is normal over $L$.

(3) There is a one to one correspondence between connected algebraic subgroups $H$ of $G$ and relatively closed intermediate fields $L$.

In (4) and (5) assume that $H$ is a connected algebraic subgroup of $G$ and $L=H^{\prime}$.

(4) $H$ is normal in $G$ if and only if $L$ is normal over $K$.

(5) Assume that $H$ is normal in $G$ and that $D$ is the Galois group of $L$ over $K$. If $N$ is any universal extension for $L$ then $I_{L}$ is normal in $I$. The homomorphisms defined by restriction and extension determine natural isomorphisms $G / H \rightarrow D \rightarrow I / I_{L}$ and the image of $G / H$ is dense in $I / I_{L}$.

Proof. Since $M$ is normal over any relatively closed intermediate field $[3$, Theorem $]$, the first three assertions follow from $[2$, Theorem 4].

Assume that $H$ is connected and normal in $G$. To show that $L$ is normal over $K$ it is sufficient to show that $L$ is stable under $G$. If $x \in L$ and $T \in G$ then for each $S \in H, T^{-1} S T \in H$. Therefore $S T x=T x$ and $T x \in L$.

Assume that $L$ is normal over $K$. Choose $N$ a universal extension of $M$ for $L$. Let $J$ be the normalizer of $I_{L}$ in $I . J$ is an algebraic matrix group [4, Lemma 4.10]. If $x \in M-L$ then, since $M$ is normal over $L$ there is an $S \in H \subseteq J$ with $S(x) \neq x$. If $x \in L-K$ then there is an $S \in D$ with $S(x) \neq x$. $S$ extends to an $\bar{S} \in I$. If $T \in I_{L}$ and $y \in L$ then $T \bar{S} y=\bar{S} y$ so $\bar{S} \in J$. Combining the above we find that the subset of $M$ left fixed 
by $J$ is $K$. By Corollary $3.2 J$ is isomorphic to an algebraic subgroup $J^{*}$ of $G^{*}$, the Galois group of $M\left(C_{N}\right)$ over $K\left(C_{N}\right)$. Since $M$ and $K\left(C_{N}\right)$ are linearly disjoint over $K$ the fixed field of $J^{*}$ is $K\left(C_{N}\right)$. Therefore $J^{*}=G^{*}, J=I$ and $I_{L}$ is normal in $I$.

The mapping of $G$ into $I / I_{L}$ defined by $S \rightarrow S I_{L}$ is clearly a homomorphism whose kernel is $L^{\prime}=H$. Therefore $H$ is normal in $G$ and the proof of (4) is complete.

If $H$ is normal in $G$ and $N$ is any universal extension for $L$ then, since $L$ is stable under $G$, the restriction mapping is a homomorphism of $G$ into $D$. The kernel is $H$, and $G / H$ is isomorphic to the subgroup of $D$ of elements which have an extension to automorphisms of $M$.

The mapping of $D$ into $I / I_{L}$ defined by $S \rightarrow \bar{S} I_{L}$ where $\bar{S}$ is any extension of $S$ to an element of $I$ is clearly well defined and an isomorphism.

The composition of these isomorphisms takes $S H$ to $S I_{L}$. A nonnull open set in $I / I_{L}$ is of the form $\left\{T I_{L}, T \in A\right\}$ where $A$ is a nonnull open set in $I$. By Proposition $3.1 G$ is dense in $I$ and $A$ contains a point of $G$. Therefore the image of $G / H$ is dense in $I / I_{L}$ and the proof is complete.

The following example shows that even if the original ground field has an algebraically closed field of constants it need not be the case that each automorphism of $L$ extends to an automorphism of $M$. This shows that the restriction mapping need not take $G$ onto $D$. In this example $L$ is itself a solution field over $K$ and $D$ is an algebraic matrix group.

EXAmple 4.2. Let $k=C(z)$ where $C$ is the complex numbers and $z$ is transcendental over $C$. We consider the classical transform which is the identity on $C$ and takes $z$ to $z+1$. Assume that $M$ is a generic solution field [2, Proposition 4] for $y_{2}=z y$ with fundamental system $(a, b)$. Set $j=a / b, e=j j_{1}$, and $f=j+j_{1}$. Then, [2, Lemmas 1 and 2, p. 511], $K=k\langle j\rangle, C_{K}=C(e, f)$ where $e$ and $f$ are algebraically independent over $C$, and for each automorphism $T$ of $M$ over $K$ there exist $x, y \in C_{K}$ with

$$
T a=x a-y e b=\left(x-y j_{1}\right) a .
$$

If $L=K\left\langle a a_{1}\right\rangle$ then $L$ is a solution field over $K$ for $y_{1}=z y$. Therefore $D$ is the full multiplicative subgroup of $C_{K}[2$, Proposition 6]. Thus there is an $S \in D$ with $S\left(a a_{1}\right)=f a a_{1}$. If there were a $T \in G$ with $T\left(a a_{1}\right)$ $=f a a_{1}$ there would be $x, y \in C_{K}$ with $x^{2}-x y f+y^{2} e=f$. The proof can now be completed by the following lemma.

LEMMA 4.3. Assume that $s$ and $t$ are algebraically independent 
over the complex numbers $C$. There do not exist $x, y \in C(s, t)$ with $x^{2}-x y s+y^{2} t=s$.

Proof. Assuming such $x$ and $y$ exist, set $w=x-y s / 2, A=t-s^{2} / 4$. Then $w^{2}+A y^{2}=s$. Write $w=a / b, y=c / d$ where $a, b, c, d \in C[s, t]$ and $(a, b)=(c, d)=1$. From $a^{2} d^{2}+A c^{2} b^{2}=s b^{2} d^{2}$, we obtain $b^{2} \mid d^{2}$ and $d^{2} \mid A b^{2}$. Since $A$ is irreducible one may multiply both $c$ and $d$ by the same constant to obtain $d^{2}=b^{2}$ or $d^{2}=A b^{2}$. In the second case $A$ $=(d / b)^{2}$ is a contradiction. If $d^{2}=b^{2}$ then $a^{2}+A c^{2}=s b^{2}$. If $c(0, t) \neq 0$ then $A(0, t)=t=(i a(0, t) / c(0, t))^{2}$ a contradiction. If $c(0, t)=0$ then $a(0, t)=0$. Since $s \mid a$ and $s|c, s| b^{2}$. Therefore $s \mid(a, b)$, the final contradiction.

\section{REFERENCES}

1. R. Cohn, Difference algebra, Wiley, New York, 1965.

2. C. Franke, Picard-Vessiot theory of linear homogeneous difference equations, Trans. Amer. Math. Soc. 108 (1963), 491-515.

3. - A note on the Galois theory of linear homogeneous difference equations, Proc. Amer. Math. Soc. 18 (1967), 548-551.

4. I. Kaplansky, $A n$ introduction to differential algebra, Hermann, Paris, 1957.

Seton Hall University 